\title{
Semantic Browsing: A New Way to Explore and Discover Heritage Treasures
}

\author{
Alan Payne \\ Deep Visuals Limited \\ B23 Science Park, Milton Road \\ Cambridge, CB4 OEY \\ alan.payne@deepvisuals.com \\ Robert Smith \\ Scott Polar Research Institute \\ Lensfield Road \\ Cambridge, CB2 1ER \\ rds39@cam.ac.uk
}

\author{
Peter Fry \\ Deep Visuals Limited \\ B23 Science Park, Milton Road \\ Cambridge, CB4 OEY \\ peter.fry@deepvisuals.com \\ Julio D'Escrivan \\ Anglia Ruskin University \\ East Road \\ Cambridge, CB2 3NH \\ julio@bitbongo.com
}

\author{
Heather Lane \\ Scott Polar Research Institute \\ Lensfield Road \\ Cambridge, CB2 1ER \\ librarian@spri.cam.ac.uk
}

\section{INTRODUCTION}

Museums and galleries have in recent years spent considerable time and effort in digitisation projects, yet the software resources available to fully explore their collections are still largely unsatisfactory. In a joint project between the new media company Deep Visuals, the Scott Polar Research Institute, and Anglia Ruskin University we are developing a new browsing system aimed at enabling museum visitors to experience more fully the museum's digital assets beyond the exhibited artefacts.

\section{SYSTEM DESCRIPTION}

The basic premise is that users should be able to explore digitised collections in a serendipitous way, much in the same manner as the keen visitor wanders through the museum space at will. To do this we have developed visual semantic browsing techniques. These allow the user to select an image on a screen and obtain a montage of related images from across the entire collection. Some of the images presented will be related in tenuous ways which motivates the user (attracted by new pictures of items they were not initially looking for) to browse further by a kind of lateral thinking process. The result is an engaging and personalised experience where the user is led through unexpected paths into the whole collection.

In addition, we have developed a number of multimedia elements that add considerably to the depth of information and the experience. One such element is the addition of audio recordings of domain experts describing their interpretation and appreciation of various parts of the collection. We have done this to capture the informal comments that an expert may make when taking a friend around the physical collection.

\section{HOW IT WORKS}

We employ Natural Language Processing (NLP) techniques to extract concepts from the textual descriptions that museums store alongside their image collections. By comparing these individual image concepts using an ontology derived from the whole collection, we are able to generate similarities between the individual items in the database. The visualisation employed is customisable based on the preference of the museum.

\section{EDUCATIONAL APPLICATIONS}

We are conducting a series of user tests with young learners between 9-11 years old to explore the use of our visual semantic browser for structuring narratives. This will assist the museum in reaching their intended learning objectives.

\section{FUTURE WORK}

We are working on a game layer as well as social networking to make museum collections accessible to broader audiences through viral means. 